\title{
Supporting information: A Criterion for the Formation of Nonequilibrium Self-Assembled
}

\section{Structures}

\author{
Andrés Arango-Restrepo, ${ }^{*, \dagger, \ddagger}$ Daniel Barragán, $₫$ and J. Miguel Rubi ${ }^{\dagger, \ddagger, \S}$ \\ $\dagger$ †epartament de Física de la Matèria Condensada, Universitat de Barcelona, Barcelona \\ $\ddagger$ Institut de Nanociencia i Nanotecnologia, Universitat de Barcelona, Barcelona \\ -Escuela de Química, Universidad Nacional de Colombia, Medellin \\ $\S$ PoreLab, Department of Physics, Norwegian University of Science and Technology, \\ Trondheim, Norway \\ E-mail: aarangor@unal.edu.co
}

\begin{abstract}
An analogous method to obtain the potential by means of the optimization of the energy required to form a structure is presented.
\end{abstract}

\section{Potential by optimization}

The energy needed to form a structure $(\Delta E)$ for a given value of $\eta$ is the sum of the energy change in the absence of dissipation and the energy dissipated in its formation, $\Delta E_{d}=T \Sigma$, where $\Sigma$ is the entropy produced: ${ }^{1}$

$$
\Delta E(\eta, t)=\Delta E_{r e v}(\eta, t)+\Delta E_{d}(\eta, t)
$$


The fact that nature evolves making efficient use of available resources, ${ }^{2,3}$ enables us to assume that an extreme value of the energy dissipated, at which $\partial \Delta E_{d} /\left.\partial \eta\right|_{\eta *}=0$, is the signature of an optimal design of the structure. Close to $\eta^{*}$, the condition fulfilled is thus:

$$
\left(\frac{d \Delta E_{d}(\eta)}{d \eta}\right)^{2} \geq \psi(\eta)
$$

where $\psi$ is a residual function measuring deviations from the optimal structure.

To minimize the energy $\Delta E$ subjected to the restriction given in Eq.(S.2), we use the method of Lagrange multipliers. We then define the Lagrangian function

$$
\mathcal{L}=\Delta E+\lambda\left(\left(\frac{d \Delta E_{d}(\eta)}{d \eta}\right)^{2}-\psi(\eta)\right)
$$

where $\lambda$ is a Lagrange multiplier. Setting $\partial \mathcal{L} / \partial \eta=0$, integrating over $\eta$ and solving for $\lambda \psi \equiv \Psi$, we obtain

$$
\Delta \Psi(\eta)=\Delta E(\eta)+\lambda\left(\frac{d \Delta E_{d}}{d \eta}\right)^{2}
$$

where the Lagrange multiplier is given by

$$
\lambda=-\left.2\left(\frac{\partial^{2} \Delta E_{d}}{\partial \eta^{2}}\right)^{-1}\right|_{\eta^{*}}
$$

One has $\lambda>0$ since the derivative is computed around the maximum of $E_{d}$.

The potential obtained in Eq.(S.4) by means of the optimization method coincides with Eq.(6) of the manuscript. 


\section{References}

(1) Arango-Restrepo, A.; Barragán, D.; Rubi, J. M. Self-assembling outside equilibrium: emergence of structures mediated by dissipation. Phys. Chem. Chem. Phys. 2019, 21, $17475-17493$.

(2) Coppens, M.-O. A nature-inspired approach to reactor and catalysis engineering. Curr. Opin. Chem. Eng. 2012, 1, $281-289$.

(3) Magnanelli, E.; Solberg, S. B. B.; Kjelstrup, S. Nature-inspired geometrical design of a chemical reactor. Chem. Eng. Res. Des. 2019, 152, $20-29$. 